\title{
An Unusual Location for Prostate Brachytherapy SeEds
}

\author{
Sivakumar Srinivasan, MD, and Sandeep Anreddy, MD
}

\section{Introduction}

Vascular migration of radioactive seeds to the lungs after prostate brachytherapy is known phenomenon. Here in we report a case of prostate brachytherapy seed migration into the right ventricle.

\section{Case Presentation}

An 83 year-old male with a past medical history significant for hypertension, hyperlipidemia, and prostate cancer status post prostate brachytherapy seed implantation was brought to the Cardiac catheterization lab for an elective catheterization for further evaluation of a recent stress test revealing antero-apical ischemia with an ejection fraction of $49 \%$. The Cardiac catheterization films are shown below (Figures 1 and 2).

\section{Discussion}

Prostate brachytherapy is achieved with radioactive seed implantation carrying iodine-125 or palladium-103. This procedure is a well-accepted therapeutic option for patients with localized prostate cancer. The small size of the seed allows for potential displacement from the periprostatic insertion site to adjacent prominent periglandular venous plexuses. The most common site of prostatic seed migration is the pulmonary vasculature barring a congenital arterio-venous malformation or congenital heart disease.

Prior reports indicate that the percentage of patients who have at least 1 seed migrate to the chest following prostate brachytherapy varies widely from $0.7 \%$ to $55 \%$, whereas the total percentage of seeds that eventually migrate is less than $1 \%$. Seed embolization to the heart is extremely rare. The true incidence rate has not been reported. Seed migration to the heart can rarely cause cardiac arrhythmias. A chest X-ray is commonly recommended following brachytherapy and during routine follow-up evaluation.

\section{References}

1. Davis BJ, Wilson TM et al., Prostate brachytherapy seed migration to the right ventricle found at autopsy following acute cardiac dysrhythmia. J Urol 2000; 164(5):1661.

2. B.J. Davis, J.F. Bresnahan and S.L. Stafford et al., Prostate brachytherapy seed migration to a coronary artery found during angiography, J Urol 2002; 168:1103.

3. H.F. Blair, A. Porter and Q.S. Chen, In vivo detection of an 125I seed located in the intracardiac region after prostate permanent brachytherapy. Int J Radiat Oncol Biol Phys 2004; 58: 888-91.

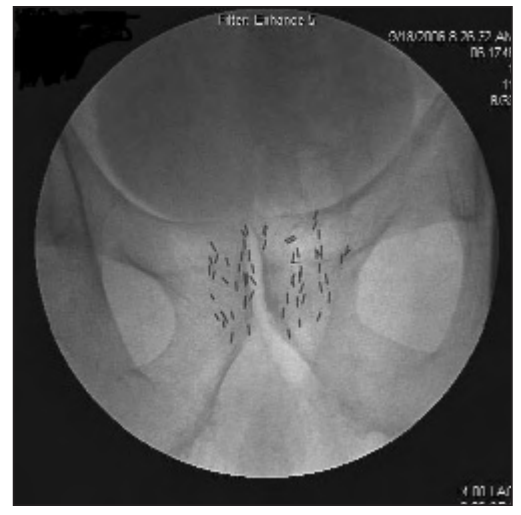

Figure 1.

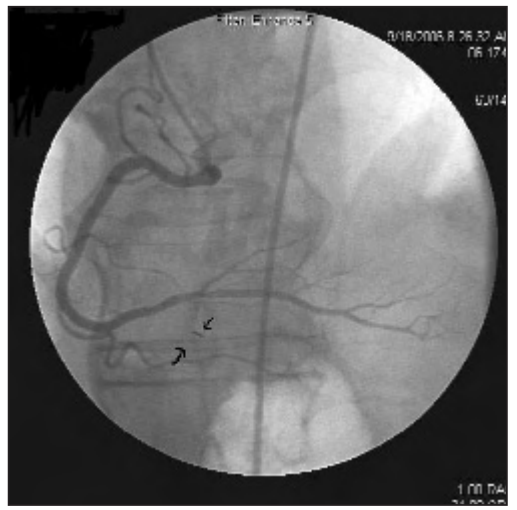

Figure 2. 